SHORT REPORT

\title{
Detection of oligoclonal free kappa chains in the absence of oligoclonal $\lg G$ in the CSF of patients with suspected multiple sclerosis
}

\author{
S Goffette, M Schluep, H Henry, T Duprez, C J M Sindic
}

J Neurol Neurosurg Psychiatry 2004;75:308-310. doi: 10.1136/jnnp.2003.010710

Background: Oligoclonal free kappa bands are present as frequently as oligoclonal lgG bands in the cerebrospinal fluid (CSF) from patients with definite multiple sclerosis (MS) and can even occur in the absence of oligoclonal lgG. As such, they too are markers of an ongoing intrathecal immune process.

Objectives: To determine how frequently oligoclonal free kappa bands are detectable in the CSF from patients with clinical signs and symptoms suggestive of MS in the absence of CSF restricted oligoclonal lgG.

Methods: An immunoaffinity mediated immunoblotting technique specific for free kappa chains was used, after isoelectric focusing of paired CSF and serum samples from 33 patients with clinical signs and symptoms suggestive of MS but without CSF oligoclonal IgG. CSF data were correlated with MRI results in the context of the new diagnostic criteria from McDonald et al.

Results: Eighteen CSF samples contained oligoclonal free kappa bands (54\%), mainly from patients with motor dysfunction (83\%) and optic neuritis (64\%). All patients with a positive MRI according to Barkhof's criteria $(n=6)$ had free kappa bands in their CSF.

Conclusions: (1) Oligoclonal free kappa bands in the CSF are related to the dissemination of MS lesions; (2) such bands should be looked for in oligoclonal lgG negative CSF, and (3) the presence of free kappa bands in the CSF may be a substitute for oligoclonal lgG in the McDonald's criteria for diagnosis of MS.

I n a previous study, ${ }^{1}$ we reported the frequent occurrence of cerebrospinal fluid (CSF) specific oligoclonal free kappa bands $(92 \%)$, and the less frequent appearance of free lambda bands (69\%), in a group of 48 patients with clinically definite multiple sclerosis (MS). In this group, all oligoclonal IgG positive CSF but one $(n=40)$ contained free kappa bands. Interestingly however, five oligoclonal IgG negative CSF samples contained free kappa bands. This observation suggested that an intrathecal immune reaction could be more sensitively detected by the presence of such bands. Indeed, free kappa bands were never present in the CSF of patients without neurological disorders $(n=26)$ and rarely detected in non-MS neurological diseases (seven out of 44 or $16 \%$ ). ${ }^{1}$ Detection of CSF specific oligoclonal IgG bands (two or more) was the topic of a consensus report ${ }^{2}$ but the value of detecting free kappa bands has not yet been widely established. ${ }^{13}$

When faced with clinical signs and symptoms suggestive of MS, neurologists use diagnostic procedures based on brain and/or spinal cord magnetic resonance imaging (MRI), and CSF analysis. The recent diagnostic criteria of McDonald et $\mathrm{al}^{4}$ refer to both techniques, and link CSF oligoclonal IgG bands and/or a high IgG index to spatial dissemination where there are two or more MRI lesions consistent with MS.

In the present study, CSF samples with no or only a single IgG band from patients presenting with clinical signs and symptoms suggestive of a demyelinating event, were specifically analysed for free kappa bands. CSF data were compared with the clinical signs and the results of MRI scans.

\section{PATIENTS}

Thirty three patients (mean age 35; range 18-63) were studied. They presented isolated clinical signs and symptoms suggestive of MS: optic neuritis $(n=11)$, objective sensory impairment $(n=7)$, motor impairment $(n=6)$, diplopia and/ or vertigo and/or ataxia $(n=8)$, neurogenic bladder $(n=1)$. Extended blood analysis and microbiological tests excluded other autoimmune, infectious, or metabolic disorders. Cerebrospinal fluid analysis showed no specific oligoclonal IgG bands $(n=29)$ or only one IgG band not present in the corresponding serum $(n=4)$. The IgG index and/or the intrathecal fraction as determined by Reiber's formula ${ }^{5}$ were normal or negative. The determination of oligoclonal free kappa chains in the serum and the CSF was done retrospectively but without knowledge of MRI results.

\section{MATERIALS AND METHODS \\ Samples}

All paired samples of CSF and serum were collected between December 1997 and September 2000 in Brussels or in Lausanne and were stored at $-80^{\circ} \mathrm{C}$. IgG concentration was determined by immunonephelometry (Beckmann Array). Routine detection of oligoclonal IgG bands was performed with slightly different techniques in both sites (affinity immunotransfer in Brussels ${ }^{1}$ and double immunostaining in Lausanne ${ }^{6}$ ). In addition, all CSF samples from Lausanne were retested in Brussels for oligoclonal IgG with identical negative results.

\section{Detection of oligoclonal free kappa bands}

Oligoclonal free kappa bands were determined by an immunoaffinity mediated capillary blotting technique as previously described. ${ }^{1}$ This technique has a detection limit of about $25 \mathrm{ng}$ of free kappa Bence-Jones protein and does not show cross reactions, neither with up to $1200 \mathrm{ng}$ of

Abbreviations: CSF, cerebrospinal fluid; MRI, magnetic resonance imaging; MS, multiple sclerosis 
polyclonal IgG, IgA, or IgM nor with free lambda bands. A possible explanation for this high specificity is the double use of the anti-free kappa antiserum, first on the polyvinyl pyrrolidone fluoride (PVDF) sheet for the immunoaffinity transfer, and then for the immunodetection after biotinylation.

\section{Magnetic resonance imaging}

Magnetic resonance imaging of the cerebrum, cerebellum, and brainstem was performed in the first 15 days after clinical onset for 26 patients and between 16 and 30 days for the seven remaining cases. If clinical signs suggested spinal cord involvement, spinal cord MRI was performed first, followed by brain MRI (four patients). Magnetic resonance images were reviewed retrospectively on the basis of the MRI criteria for MS from Barkhof et al ${ }^{7}$ and Tintore et $a l_{,}^{8}$ as recommended by McDonald et al, ${ }^{4}$ although intravenous gadolinium DTPA was performed in only 17 of the 33 patients (51\%). According to Barkof's criteria, MRI is considered as positive for MS when three of the four following are present: (1) one Gadolinium (Gd) enhancing lesion or nine $\mathrm{T} 2$ hyperintense lesions if no Gd enhancing lesion; (2) one or more infratentorial lesions; (3) one or more juxtacortical lesions; (4) three or more periventricular lesions. For each of these items, a spinal cord lesion can substitute for a brain lesion. If these criteria were not fulfilled, but two or more MRI lesions consistent with MS were observed, we used the term "suggestive MRI". Normal MRIs or non-specific lesions (a single lesion or a few millimetric lesions) were considered as unhelpful for the diagnosis of MS.

\section{RESULTS}

According to study selection criteria, no CSF samples contained oligoclonal IgG bands and four contained only one IgG band not present in the corresponding serum. In contrast, in $18(54 \%)$ of the 33 patients, two or more oligoclonal free kappa bands were observed in the CSF and not in the paired serum (fig 1). The kappa bands appeared most frequently in the CSF from patients with motor impairment $(5 / 6$ or $83 \%)$ followed by those with optic neuritis (7/11 or $64 \%)$, and sensory impairment (3/7 or $43 \%$ ). The CSF from patients with diplopia, vertigo, or ataxia rarely showed free kappa bands ( $2 / 8$ or $25 \%)$. Cerebrospinal fluid from the patient with isolated neurogenic bladder also showed free kappa bands. Of note, three of the four CSF samples with one single IgG band contained oligoclonal free kappa bands.

Magnetic resonance imaging was positive for MS in only six cases and free kappa bands were present in all six cases (table 1). Two or more suggestive MRI lesions were found in 11 patients and the CSF from seven of these (64\%) contained free kappa bands. Non-specific or normal brain MRI findings were observed in 16 patients of which four presented at least one spinal cord lesion; only five of these 16 (31\%) displayed free kappa bands in the CSF and one had a single IgG band without free kappa chains.

\section{DISCUSSION}

In a previous study, ${ }^{1}$ we found a high occurrence (92\%) of free kappa bands in the CSF from MS patients, and not in their serum. Their presence was significantly correlated with their CSF concentrations; ${ }^{9}$ their absence in the corresponding sera is the result of a dilution effect in blood volume. In addition, the high renal clearance of low molecular weight proteins concentrates them in the urine, and increased amounts of electrophoretically homogenous free light chains have been detected in the urine of certain patients. ${ }^{10}$
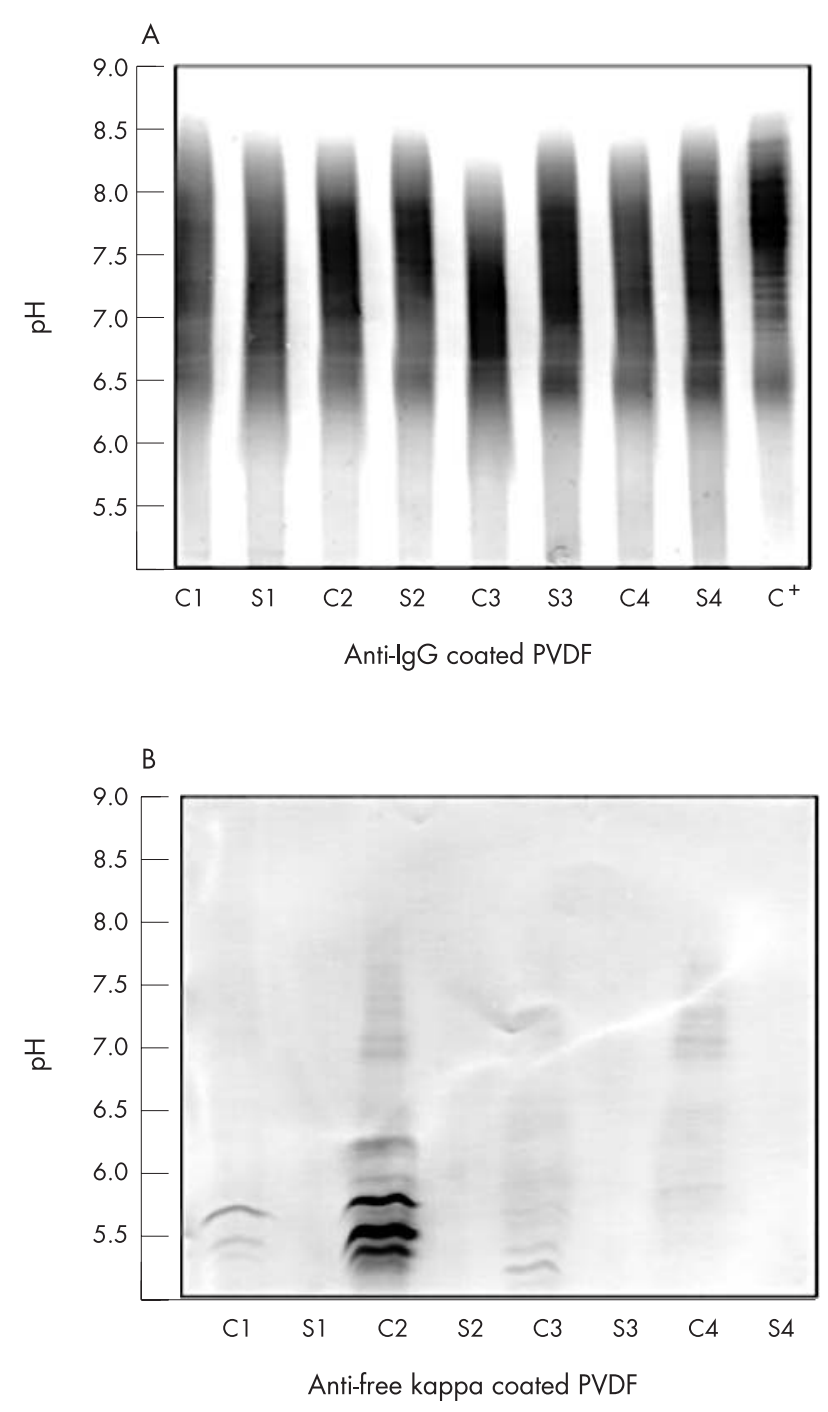

Figure 1 Immunoblots of $\operatorname{lgG}(\mathrm{A})$ and of free kappa bands (B) from four unconcentrated cerebrospinal fluids (CSF) and their corresponding sera (S), diluted to the same lgG concentration, after simultaneous isoelectric focusing in agarose gel. In spite of the absence of clearly detectable oligoclonal $\lg G$ bands, note the presence of several free kappa bands restricted to the CSF and their complete absence in the corresponding sera. The last sample $\left(\mathrm{C}^{+}\right.$, top right) is an oligoclonal positive control CSF.

It is unlikely that the presence of free kappa bands is related to the degradation of immunoglobulins, as free heavy chains $(\gamma, \alpha, \mu)$ are never found in the CSF. ${ }^{11}$ In contrast, excess free light chains have been detected in sera from patients with increased synthesis of immunoglobulins, as observed in chronic leukaemia, multiple myeloma, and immune disorders, such as Sjögren's syndrome.

We carefully selected patients with clinical signs and symptoms suggestive of MS, but without CSF restricted oligoclonal IgG as defined in the European consensus paper. ${ }^{2}$ Four of these patients did, however, display a single abnormal IgG band without a corresponding band in the serum; the clinical significance of this finding remains elusive. ${ }^{12}$ We looked for the presence of free kappa bands in the CSF, as these are more specific for MS than lambda bands, as shown in previous studies by us ${ }^{1}$ and others. ${ }^{13}$ In addition, kappa/lambda ratios of CSF IgG from MS patients have been repeatedly shown higher than those of normal controls. ${ }^{14}$ 
Table 1 Results of initial magnetic resonance imaging in the different cerebrospinal fluid patterns

\begin{tabular}{llll}
\hline Patients $(n=33)$ & $\begin{array}{l}\text { Positive } \\
\text { MRI* }\end{array}$ & $\begin{array}{l}\text { MS suggestive } \\
\text { MRI† }\end{array}$ & $\begin{array}{l}\text { Non-specific or } \\
\text { normal brain MRI }\end{array}$ \\
\hline $\lg G(-) / \kappa+(n=15)$ & 5 & 6 & 4 \\
$\lg G(1$ band $) /$ & 1 & 1 & $1 \neq$ \\
$\kappa+(n=3)$ & & 0 & 1 \\
$\lg G(1$ band $) / \kappa(-)$ & 0 & 4 & $10 \S$ \\
$(n=1)$ & 0 & 11 & 16 \\
$\lg G(-) / \kappa(-)(n=14)$ & 6 & &
\end{tabular}

*According to the strict criteria of Barkhof et $\mathrm{al}^{7}$ (a spinal cord lesion can substitute for a brain lesion).

†Two or more lesions suggestive of MS either in the brain or in the spinal cord.

$\ddagger$ This patient had only a single spinal cord lesion.

$\S$ One of these 10 patients had only a single spinal cord lesion.

$\chi^{2}$ (positive or suggestive MRI $v$ aspecific or normal MRI; positive $v$ negative free kappa) with Yate's correction: $p=0.0239$.

Detection of free light chains has at least three theoretical advantages over IgG detection: (1) because of a lower molecular weight, free light chains may diffuse more easily into the subarachnoid space; (2) IgG molecules may be bound either to their specific antigen(s) via their $F\left(a b^{\prime}\right) 2$ fragments or to myelin or cellular plasma membranes via their Fc fragments within the nervous tissue; (3) free light chains are more easily detectable by immunoblotting techniques than IgG bands, because of the absence of a polyclonal background.

Free kappa bands were detected in 18 of 33 patients, mostly in those with motor impairment $(5 / 6,83 \%)$ and with optic neuritis $(7 / 11,64 \%)$, this latter figure being similar to what we observed in a cohort of 27 patients with optic neuritis (55\%). ${ }^{15}$ The most striking finding, however, was the correlation between the occurrence of free kappa bands and the presence of MRI abnormalities. All patients with a positive MRI, as defined by Barkhof's criteria, ${ }^{7}$ displayed free kappa bands, as did seven of the 11 patients $(64 \%)$ with a suggestive MRI. This percentage dropped to 31 (5/16) in patients with normal or non-specific MRI scans. In addition, three of the four patients with a single IgG band had CSF free kappa bands, indicating that the finding of a single IgG band is worth noting and has an immunological relevance.

We can conclude, therefore, that (1) the presence of free kappa bands in the CSF is related to the dissemination in space of the MS lesions; (2) free kappa bands should be looked for when CSF is oligoclonal IgG-negative, and (3) free kappa bands in the CSF may be substituted for oligoclonal IgG in the new McDonald criteria for diagnosis of MS.

\section{ACKNOWLEDGEMENTS}

The authors are grateful to Mrs Marie-Paule Van Antwerpen and to Mrs Elodie Allaz for excellent technical assistance. This work was supported by a grant from the Belgian Charcot Foundation.

\section{Authors' affiliations}

S Goffette, Service de Neurologie, Cliniques universitaires Saint Luc, UCL, Brussels, Belgium

C J M Sindic, Laboratoire de Neurochimie et Cliniques, universitaires Saint Luc, UCL, Brussels, Belgium

M Schluep, Service de Neurologie et Centre Hospitalier Universitaire Vaudois, Lausanne, Switzerland

T Duprez, Département d'Imagerie, Cliniques universitaires Saint Luc, UCL, Brussels, Belgium

H Henry, Laboratoire de Chimie clinique, Centre Hospitalier Universitaire Vaudois, Lausanne, Switzerland

Competing interests: none declared

Correspondence to: Professor C J M Sindic, Laboratoire de

Neurochimie, Université Catholique de Louvain, 53-59 Avenue Mounier, 1200 Brussels, Belgium; sindic@nchm.ucl.ac.be

Received 1 February 2003

In revised form 1 May 2003

Accepted 17 June 2003

\section{REFERENCES}

1 Sindic CJ, Laterre EC. Oligoclonal free kappa and lambda bands in the cerebrospinal fluid of patients with multiple sclerosis and other neurological diseases. An immunoaffinity-mediated capillary blot study. J Neuroimmunol 1991;33:63-72.

2 Andersson M, Alvarez CJ, Bernardi G, et al. Cerebrospinal fluid in the diagnosis of multiple sclerosis: a consensus report. J Neurol Neurosurg Psychiatr 1994;57:897-902.

3 Gallo $\mathbf{P}$, Tavolato B, Bergenbrant $S$, et al. Immunoglobulin light chain patterns in the cerebrospinal fluid: a study with special reference to the occurrence of free light chains in cerebrospinal fluid with and without oligoclonal immunoglobulin. G J Neurol Sci 1989;94:241-53.

4 Mc Donald WI, Compston A, Edan G, et al. Recommended diagnostic criteria for multiple sclerosis: guidelines from the International Panel on the diagnosis of Multiple Sclerosis. Ann Neurol 2001;50:121-7.

5 Reiber $\mathrm{H}$. The hyperbolic function: a mathematic solution of the protein flux/ CSF flow model for blood-CSF barrier function. J Neurol Sci 1994:126:243-5.

6 Keir G, Luxton RW, Thompson EJ. Isoelectric focusing of cerebrospinal fluid immunoglobulin G: an annoted update. Ann Clin Biochem 1990;27:436-43.

7 Barkhof F, Filippi M, Miller DH, et al. Comparison of MRI criteria at first presentation to predict conversion to clinically definite multiple sclerosis. Brain 1997:120:2059-69

8 Tintore M, Rovira A, Martinez MJ, et al. Isolated demyelinating syndromes: comparison of different MR imaging criteria to predict conversion to clinically definite multiple sclerosis. Am J Neuroradiol 2000;21:702-6.

9 Fagnart OC, Sindic CJM, Laterre EC. Free kappa and lambda light chain levels in the cerebrospinal fluid of patients with multiple sclerosis and other neurological diseases. J Neuroimmunol 1988;19:119-32.

10 Mehta PD, Cook SD, Coyle PK, et al. Free light chains in multiple sclerosis urine. Mult Scler 1998:4:254-6.

11 Gallo P, Piccinno MG, De Rossi A, et al. Free light chains of immunoglobulins in the cerebrospinal fluid of human immunodeficiency virus type 1-infected patients. J Neuroimmunol 1990;26:229-38.

12 Ben-Hur T, Abramsky O, River Y. The clinical significance of a single abnormal immunoglobulin band in cerebrospinal fluid electrophoresis. J Neurol Sci 1996;136:159-61.

13 Krakauer M, Nielsen HS, Jensen J, et al. Intrathecal synthesis of free immunoglobulin light chains in multiple sclerosis. Acta Neurol Scand 1998;98:161-5.

14 Aragu S, Kagimoto H, Wada A, et al. Kappa/lambda ratios in lgG, IgA and $\lg M$ of cerebrospinal fluid and of sera in patients with multiple sclerosis. Autoimmunity 1989;5:133-7.

15 Frederiksen JL, Sindic CJM. Intrathecal synthesis of virus-specific oligoclonal $\operatorname{lgG}$, and of free kappa and free lambda oligoclonal bands in acute monosymptomatic optic neuritis. Comparison with brain MRI. Multiple Sclerosis 1998;4:22-6. 